\title{
Protée
}

\section{Le corps selon Duchamp}

\section{Herman Parret}

Volume 28, numéro 3, 2000

Mélancolie entre les arts

URI : https://id.erudit.org/iderudit/030608ar

DOI : https://doi.org/10.7202/030608ar

Aller au sommaire du numéro

\section{Éditeur(s)}

Département des arts et lettres - Université du Québec à Chicoutimi

\section{ISSN}

0300-3523 (imprimé)

1708-2307 (numérique)

Découvrir la revue

\section{Citer cet article}

Parret, H. (2000). Le corps selon Duchamp. Protée, 28(3), 88-100. https://doi.org/10.7202/030608ar

\section{Résumé de l'article}

Duchamp est glorifié comme le fondateur de l'art conceptuel. Toutefois, l'arsenal duchampien est rempli avant tout de concepts-corps. La pratique duchampienne met en scène le concept de corps essentiel, de sa mécanique, de ses fragments, de ses indices. Il se révèle impossible de penser le corps duchampien sans prendre en compte la nécessité de l'empreinte. La " gloire des empreintes » introduit une topologie de l'infra-mince, thème que Duchamp va développer tout au long de ses Notes. d'utilisation que vous pouvez consulter en ligne.

https://apropos.erudit.org/fr/usagers/politique-dutilisation/ 


\section{Hors dossier}

À propos de Marcel Duchamp 


\title{
LE CORPS SELON DUCHAMP
}

\author{
H ERMAN PARRET
}

L'érotisme de précision de la machine

Fragments de chair essentiels

Indices fautifs et prothèse agonistique

Gloire des empreintes
Q ue M arcel D uchamp soit l'iconoclaste et le fourvoyeur des valeurs modernistes, qu'il soit l'éclaireur de l'art contemporain, personne n'en doute. La pratique du ready-made a problématisé la notion même d'œuvre d'art tout comme la fonction d'artiste, et on ne s'est pas encore remis de ce dur coup subversif qui a mis un point à tant de certitudes modernistes ${ }^{1}$. Sous un angle plus constructif, D uchamp est glorifié comme le fondateur de l'art conceptuel, et c'est le ready-made qui ferait de I'art uneapologiedu concept. U rinoir, goutte-bouteilles, porte-manteaux, pelle, roue de bicyclette, tant de concepts-objets dont notre imaginaire fin de millénaire ne pourra plus jamais se défaire. Toutefois, I'arsenal duchampien est rempli avant tout de concepts-corps, et c'est le corps que D uchamp conceptualise dès 1909, l'année où il peint en impressionniste et avec tendresse le portrait de sa sœur Y vonne. II analyse en 1911, en cubiste cette fois-ci, le mouvement du N u descendant un escalier, pour laisser définitivement la peinture derrière lui vers 1914, geste théorique et pratique radicalement subversif, souvent commenté2. D uchamp s'immerge ensuite dans l'iconographie de La M ariée mise à nu par ses célibataires, même (1915-23). A près avoir vécu sans voix d'artiste pendant plusieurs décennies, D uchamp travaillera pendant vingt ans et dans le plus grand secret au concept radical de corps-corps, ce corps de femme aux membres amputés dont la masse de chair se construit autour d'une vulve rasée et abyssale: Étant donnés: $1^{\circ}$ la chute d'eau, $2^{\circ}$ le gaz d'éclairage (1946-1966).

Le corps selon D uchamp ne se laisse prédiquer d'aucune catégorie esthétique - le corps n'est ni beau, ni sublime, ni gracieux, ni dégoûtant non plus-, aucune intérioriténe s'y manifeste, aucunephénoménologien'y découvrira jamais quelque signifiance. II s'agit en fait du corps essentiel, le corps marqué par le sexe et la mort, par É roset Thanatos, et par rien d'autre. Q uand C abanne questionne $D$ uchamp sur le rôle de l'érotisme dans son œuvre, il répond: «Énorme. Visible ou sous-jacent, partout», et D uchamp énonce à J ouffroy que le sexe est la seule chose qu'il prend vraiment au sérieux. II est vrai que l'énergie érotique déborde les catégories sexuelles conformes, restrictives et répressives. D uchamp souligne avec insistance que l'«on est toujours à l'étroit dans son sexe», qu'Éros a plus de souffle ${ }^{3}$. Le sexe éclate dans toute la création (artistique, divine), et pourtant Duchamp prêche une certaine distance, voire une certaine modération. Cet effet de distanciation est créé par les jeux de mots et cette masse fourmillante de notes, fabuleuses à foison, à peine interprétables, de la Boîte verte de 1934 et dela Boîteblanche de 1966 ensuite. Ces fragments textuels ont un effet de distanciation, ilscréent 
une certaine indifférence à l'égard de ce sérieux essentiel qu'est le sexe, par leur ton d'humour, d'ironie, d'allégresse même. Pour D uchamp d'ailleurs, lesexeet la mort n'ont rien de tragique, au contraire «Éros, c'est la vie», et en matière de sexe et de mort, nous renseignele doux sourire de M arcel, «il n'y a pas de solution puisqu'il n'y a pas de problème». Et pourtant l'iconographie duchampienne des corps n'est pas euphorisante du tout: ce sont des corps androgynes - Rrose Sélavy-, corps mécaniques, tout en tuyaux et en trompes, corpsfragmentarisés, corps impui ssants de sexe et de mort, corps qui s'écoulent en difformité, corps «prothétisés». C'est bien de ce corpslà, dont $\mathrm{D}$ uchamp nous livre le concept, de ce corps essentiel, que nous nous permettons d'évoquer la figuration.

\section{L'érotisme de précision de la machine}

Duchamp vit à partir de 1912 le fantasme de la «grande machine». Le «cubisme viscéral» reste encore pour deux ans la contrainte lui permettant de focaliser la thématiquequi s'accomplira ensuite dans Le G rand V erre. N ous nous limitonsà la $M$ ariée et son érotismemécanique de précision. Le $\mathrm{Nu}$ descendant un escalier thématise l'érotismedelanuditétandisquelaVierge[ill. 1], esquisse préliminaire pour Le Grand Verre, connote par son titre même l'univers explicitement sexuel. La Femme Artificielle s'installe, dans sa féminité, dans son artificialité. D ans Le Passage de la Vierge à la M ariée [ill. 2], le «tubisme» de Léger devient bien influent. Biologique, gyné cologique, le corps defemmedevient un amalgamed'élé ments mécaniques et de surfaces abstraites, en mouvement vers la droite, donc «en passage». Passage, comme route anatomique d'une vierge vers une mariée, passage initiatique, mystique, vers la féminité accomplie. On aboutit ainsi à la figuration de la $M$ ariée [ill. 3], toujours de 1912, qui s'installera deux ans plus tard et définitivement dans Le G rand Verre. C ette M ariée est moins baroque, plus schématique, plus compacte que la Vierge qui n'était qu'«en passage». Le désir d'homme, le regard voyeuriste est invité par $\mathrm{D}$ uchamp à disséquer avec une précision diagrammatique la machine cadavérique de la Femme et il y découvre ses secrets intimes, des organes viscéraux reliés par des tubes, trompes et cylindres. L'archétype mystique de la Féminité, l'Autre objectivé du regard masculin, n'est rien qu'un moteur alchimique. La M ariée du G rand Verre[ill. 4] est l'aboutissement par schématisation, par épuration, rien que les organes essentiels pour le fonctionnement essentiel, celui d'aimer pour mourir. Duchamp a souvent analysé en détail le statut de la M ariée et décrit in extenso sa géométrie ${ }^{4}$. La M ariée, on le verra dans la suite, l'a fasciné et obsédé jusqu'à la fin de ses jours.

La M ariée miseà nu par ses céli bataires, même raconte leV oyage du gaz d'éclairage, voyageà tâtons tout au long des artères de cettemachineà fairel'amour. Jaillissement des liquides gazeux, transportés, retardés, accélérés par le M oulin à eau, par la Broyeuse de chocolat, éparpillés par les $G$ rands ciseaux, recueillis par la Baratte ventilateur. Q uelle impuissance dans le passage vers le domaine de la M ariée! Le contact avec I'Autre n'est pas de tact mais de regard. La flèche lestée du voyage du gaz d'éclairage se heurte aux T ableaux d'oculiste, et il ne restequela Lentille de Kodak pour contempler le désir de la M ariée. Comme les Célibataires, la M ariée elle aussi est tout épanouissement. Sa mécanique complexe est solidement tenueen équilibre par la Guêpe et la Girouette, en bas, et elle produit par amour - comme unemèrelelait amer deson sein - laV oix lactéechair, nébuleuse désireuse, généreuse, langage de la M ariée qui ne sera pas écouté, appel pour que le gaz d'éclairage touche et provoque l'orgasme vaporisant, explose dans la découverte de la quatrième dimension. Duchamp croyait à cette quatrième dimension, cet espace supérieur transcendant l'ennui des illusions de la perception sensorielle, espace de l'épanouissement des corps. M ais avant tout, D uchamp se référait à son G rand Verre comme à «cette grande saloperie», cette métaphore érotico-mécanique, cette iconologie sans honte de l'amour, cette spéculation a-sentimentaledu passage delaV iergeà la M ariée, del'opération voulue mais non réussie de dépucelage.

Scrutons un instant la figure de la FemmeArtificielle. Son organe principal est le Pendu femelle rattachéen bas par la guêpe ou le cylindre-sexe et par la girouette. Allongée, nue - dénudée par l'insistance des regards des célibataires-, la M ariée est un petit moteur autonome dont les besoins sont alimentés par son propre parfum d'amour - essence d'amour-, par les étincelles de son magnéto-désir. Ce parfum, ces étincelles sont produites dans la G uêpe et transportées dans le Pendu Femelle, 
beaucoup plus mobile que la partie correspondante du tableau de 1912 - il semble même que le Pendu Femelle, rien que légèrement attaché, peut tourner en rond sous la pression de l'essence d'amour. Tête et corps sont visibles, tout comme dans le tableau de 1912, avec cette différence que le Pendu Femelle commande la machine qui «crache» la V oie lactée.

Le dépositaire sémantique de ce programme iconographique est vaste et ouvert, et maints interprétants peuvent être construits en toute compatibilité. Phénoménologiquement, la $M$ ariée n'est pas séduisante, et passablement absurde. Elle reste une inconnue, un hiéroglyphe à déchiffrer. Est-ce un fossile, le squelette d'un oiseau? Forme humaine quand même puisqu'elle est suspendue par un anneau - anneau nuptial, pourrait-on se demander - à un crochet, seule figuration réaliste dans le domaine de la $M$ ariée? Insecte - excellente métaphore de l'inexorabilité de la pulsion sexuelle-, pantin, marionnette, pendue bien vivante puisque inspirée par le souffle gazeux que son cylindre-sexe génère, et qu'elle transmettra par des pulsations de jouissance à la V oie lactéechair. Beautéimpersonnelleet pale de déesse lunaire autoritaire, sortedeSalammbô, d'H érodiade, deSalomé, sorte de cygne mallarméen dans Le Vierge, le vivace et le bel aujourd'hui, digne et lucide, sachant que son désir «évaporisant » cache mal sa frigidité.

Structuralement, le domaine de la M ariée est plus confus, plus diffus que celui descélibataires. Comprenant plus delignes obliques, plus de courbes, le domaine dela $M$ ariée est plus biologique que géométrique, plus organique sans doute quemécanique. D uchamp dans ses notes l'avait énoncé ainsi :

La M ariée à sa base est un moteur. $M$ ais avant d'être un moteur qui transmet sa puissance timide - elle est cette puissancetimidemême. Cette pui ssancetimideest une sorte d'automobiline, essence d'amour, qui, distribuée aux cylindres bien faibles, à la portée des étincelles de sa vie constante, sert à l'épanouissement de cette vierge arrivéeau terme de son désir. ${ }^{5}$

Par conséquent, la puissance timide est vitale et biologique avant de se transposer dans le mécanique. Le déshabillement de la M ariée dénude jusqu'au corps interne et nous dévoile les contractions viscérales des organes. La Boîte verte confirme l'intérêt de Duchamp pour les machines, machines à vapeur, machines à fermentation, machines électriques, machines optiques également. Vapeur, fermentation, étincelles électriques, projection optique, en effet, guident, dans Le G rand V erre, leV oyage du gaz d'éclairage. $M$ ais $D$ uchamp est autant fasciné par la dissection anatomiqueet par la radiographie des organes internes - il prétend que la radiographie de deux corps pendant la copulation procure une photo instantanée de la quatrième dimension...

D uchamp feuilletait souvent les catalogues d'instruments médicaux, surtout desinstruments gynécologiques, et il est certain qu'il a médité sur la valve Auvard [ill. 5], présentée dans le catalogue $\mathrm{H}$ artmann de 19116 , instrument qui, en s'insérant dansl'utérus, réal ise le plusgrand degré de proximité avec l'essentiel. Réconciliation, par conséquent, du mécanique et du biologique, glissement métonymique étourdissant du contenu vers le contenant. $N$ e réprimons pas en cet instant cette découverteinnommable. LePendu Femelleest une valve Auvard, et la figuration duchampienne de la Femme Artificielle est ainsi l'interface du biologique contenant et du mécaniquecontenu, interface de l'utérus moulant la valve Auvard. Le dessin que Duchamp réalise en février 1968 [ill.6], quelques mois avant sa mort, nous montre sa dernière M ariée, enveloppée dans son halo fantasmatique, ce Pendu Femelle tout en chair cette fois. C ontinuitéfigurative d'une consistance étonnante: de la valve Auvard au Pendu Femelle, du Pendu Femelle à la M ariée qu'il amène avec lui dans la mort.

Le récit du Voyage du gaz d'éclairage met en scène une fabuleuse tragédie naturelle, mais une tragédie qui devrait nous inciter à l'allégresse, non aux pleurs. D uchamp, comme le voulait $\mathrm{N}$ ietzsche, dit OUI à la vie. É panO U I ssement, éblO U I ssement, jo U I ssance, au cœur de ces trois mots typiquement duchampiens, il y a lemot de la fin: O UI ${ }^{7}$. N i pessimiste, ni optimiste, la mise en scène du Grand Verre nous confronte avec le rite de l'amour, le besoin d'épanouissement des Célibataires et de la $M$ ariée, le besoin de décharge de la semence, ce «gaz d'éclairage», en bas du Grand Verre, le besoin d'évaporation, de la lactification généreuse, en haut, semence et lait, liquides sacrifiés dans le rite de l'amour, sacrifiés en vain peut-être, et pourtant pas, utile quand même quand il faut apprendreà mourir tous les jours de sa vie. 


\section{Fragments de chair essentiels}

II va sans dire que le corps selon D uchamp ne doit rien au canon esthétique classique. C e n'est pas le corps de «la vie moderne» non plus, comme chez $\mathrm{M}$ anet, ni le corps «existentiel» ou le corps vécu de l'intérieur. C'est bien plutôt le corps sur la table de dissection, sous l'œil du chirurgien, du gynécologue, toujours «sousl'œil de», des témoins oculaires dans Le Grand Verre, du voyeur qui perceson regard libidinal àtravers les deux minuscules trous de la lourde porte qui rend la femme de Étant donnés..., qui se livre en peepshow, pour toujours intouchable. Étudions ces fragments de chair essentiels. D éjà en 1910, le Portrait du D octeur D umouchel [ill. 7], grande réussite fauviste, inquiète par une particularité: des irisations entourent le visage comme un nimbe entoure la tête d'un saint, et surtout elles émanent de sa main gauche aux doigts écartés comme si eux-mêmes étai ent la source de ce rayonnement. LeBuisson, de 1911, recréece phénomène dehalo quel'on va d'ailleurstrouvé présent jusque dans La M ariée mise à nu... de 1968 que I'on vient de contempler. D uchamp déclarait: «Le halo autour de la main [du D octeur D umouchel] [...] est un signe de mes préoccupations subconscientes vers un métaréal isme»8. Si l'irisation mauve de la main de Dumouchel est motivéepar un geste antinaturaliste et par la défensed'un art non rétinien, il est vrai que figural ement cetteirisation a comme effet la fragmentarisation du corps, I'amputation, pour ainsi dire, de la main. La focalisation de la main, à cause de cette irisation, provoque la supposition d'une coupure. De la main au pied, de 1910 à 1959, ce pied cette fois-ci est réellement amputé et enfermé dans sa boîte de plastique. Cette nature morte horrifiante qu'est T orture morte [ill. 8] est une plante de pied, peu visible en général, à peine socialisable. Le pied signifie le contact de I'homme avec la terre, aucun bonheur promis ici, rien quel'appétit de la vermine et la puanteur de la mort.

À la manière de Delvaux [ill. 13], collage de 1942, montre, dans un jeu spéculaire, des seins, fragment de la $M$ ariée en chair présente dans son absence. C es seins, en effet, sont pris dans une lunette, seins lunaires, sur le fond noir d'une nuit planétaire, éclairés par un soleil frontal tout rond. Et ce fragment de $M$ ariée déploie même sa V oie lactée, ce ruban plié: les seins lunaires sont ainsi offerts en cadeau aux C élibataires. Cinq ans plus tard, D uchamp fabrique plusieurs plâtres de sein - M aria $M$ artins, femme de l'ambassadeur argentin à $\mathrm{N}$ ew Y ork et maîtresse de D uchamp, sert à cetteépoque de modèle pour Étant donnés... et également pour ces plâtres de sein; M aria M artins qui, dans la panoplie féminine peuplant la vie de D uchamp, a incarné sans aucun doute le plus parfaitement le fantasme de la M ariée. La couverture du catalogue del'Exposition Internationale du Surréalisme de 1947 reprend Prière de toucher [ill. 9]: sur un fond noir, fabriquéen caoutchouc mousse, en trois dimensions et de grandeur nature, coloré, ce fragment de corps exalteune poétique sexuelle qui nous mène tout doucement vers l'essentiel, la vulve de la M ariée.

Le même sein se retrouve sur le corps, amputé de sa tête et de ses membres, dans une étude préliminaire de Étant donnés... [ill. 14 ], de 1948-49, étude intitulée Le gaz d'éclairage et la chute d'eau. La M ariée est enfin intégralement dévoilée, non plus dans la quatrième dimension, invisibleet projetée dans $L$ eG rand Verre, mais dans lestrois dimensions bien réelles d'une «installation», qui n'en est pas vraiment une puisqu'on ne peut la contempler que d'un seul point de vue, dans une seule perspective, celle du voyeur ${ }^{9}$. L'érotisme chez D uchamp est entêtant, cérébral, obsessionnel. Étant donnés... s'organise tout entier autour du sexe béant, rasé, glabre d'une femme allongée comme après l'orgasme. Étant donnés..., il est vrai, tourneen dérision L'O riginedu monde de Courbet et «cette perruque de blond sale» qui couvre le ventre de la femme exposée dans ce tableau. Comparé à L'O riginedu monde, Étant donnés.. ajoutela profondeur comme troisième dimension et offre un surcroît de visibilité: la lumière est trop intense et la chair trop grenue. D 'aprèsune description de Jean C lair, dansÉtant donnés... «[le corps] apparaît comme une enveloppe sans intérieur, une carcassevide, un mouleen creux, unecoque sans chair, une pellicule, un leurre» 10 . Un seule main est visible: elle brandit un Bec Auer incandescent et phallique, seule présence mâle, ce gaz d'éclairage fécondant maistenu loin du sexe, non-rencontreencoretout comme au temps du Grand Verre, lampe tenue encore pour illuminer maximalement I'indéniable punctum ${ }^{11}$.

Duchamp produit en 1950, en pleine période de préparation de Étant donnés..., une sculpture en plâtre galvanisé. Feuilledevignefemelle[ill. 10] est unesculpture 
moulée à partir d'un sexe féminin réel, et elle sera photographiée pour la couverture du livre intitulé Le Surréalisme, même 1 d'AndréBreton, en 1956. Cet objet est l'empreinte d'une aine féminine, un moule qui s'applique sur les pudenda féminins comme ces feuilles de vigne jadis appliquées sur le sexe d'Apollon. 0 bjet$D$ ard [ill.11] - le jeu homonymique est remarquableest le contrepied de la Feuille de vigne femelle, ou pas? En tout cas, c'est une empreinte également, mais plutôt un moulage qu'un moule. Objet-D ard n'est sans doute pas une fantaisie phallique mais un moule intime et profond de l'organe féminin, son relevé minutieux ${ }^{12}$. Autre valve Auvard, en conséquence. D uchamp insiste ainsi sur la réversibilité des organes femelle et mâle. 0 bjet$D$ ard a effectivement une apparence phallique mais en fait il s'agit plutôt d'une structure en doigt de gant retourné. D 'ailleurs, ce tube pseudo-phalliques'infléchit curieusement et, comme dans la topologie dela Bouteille de K lein que $\mathrm{D}$ uchamp connaissait très bien, «on peut prolonger cet infléchissement en imagination jusqu'à le faire pénétrer dans l'espèce de racine dont il est issu $\gg 13$. L'hermaphrodisme a toujours tenté D uchamp, il suffit de penser à son jeu sur le travesti : $M$ arcel D uchamp est R rose Sélavy, la verge est le moule de la vulve, topologie duchampienne abolissant la différence sexuelle14 .

T opologie des passages infinitésimaux, des différences infra-minces - le terme infra-mince est chéri par Duchamp 15. Vulve et verge sont des identiques à intervalleinfra-mince, commedesjumeaux, commedeux gouttes d'eau. Les $\mathrm{N}$ otes de D uchamp comportent un chapitre entier sur Infra-mince16, avec une série désordonnée d'exemples parfois assez éclairants. D uchamp s'y demandequel est le rapport del'infra-mince avec le principe d'identité. L'infra-mince est dans la réflexion du miroir ou de verre, dans la transparence: «peinturesur verrevuedu côténon peint donneun inframince». $\mathrm{O}$ u encore: «la différence entre deux objets faits en série (sortis du mêmemoule) est un infra-mincequand le maximum de précision est obtenu». N on seulement la transparence génère l'infra-mince mais le toucher également. «C aresses infra-minces»: dansle domaine du toucher («planer à fleur d'uneautre surface: on passe par desmomentsinfra-minces»), commed'ailleurs dans celui des odeurs («odeurs plusinfra-minces queles couleurs»), la «différence séparative» est infra-mince. G eorges D idi-
H uberman considèrel'hypothèse del'infra-mincecomme le véritable portant théorique de l'œuvre entière ${ }^{17}$, et il constateque cettehypothèse fait convertir chez D uchamp aisément l'optique vers le tactique. «C ontact et inframince» avait notéD uchamp: I'infra-mince, presquenon identifiable, est avant tout un écart tactile, une possibilité physiquesubliminale. L'hypothèse del'infra-mince, il est vrai, semble reposer sur une phénoménologie de l'affleurement. Si c'est vrai quel'écart visuel fait signe vers le contact, il est vrai aussi que dans le toucher il n'y aura jamais identité de deux objets: l'écart infra-mince subsiste même «s'il y a une approximation pratique de la similarité». Pas la perfection du même, pas de répétition, mais seule la ressemblance qui marque les produits de reproduction (D uchamp distingue bien clairement entre répétition et reproduction).

Et surtout, pour D uchamp, la topologie de l'inframince marque et génère l'érotisme: I'érotisme est la force identitaire dela différenceinfra-mince. Lecaractèreinframince de l'approximation est celui de la caresse L'approximation est progressive ${ }^{18}$ : elle peut être inchoative mais également optimalecomme dansl'emboîtement ou I'interpénétration. C'est le cas de Coin de chasteté [ill. 15], œuvre en plâtre galvanisé et plastique dentaire, de 1954. Coin de chasteté représente précisément l'enchâssement de la F euille de vigne femelle(1950) et de l'O bjetD ard (1951), ces deux objets jumeaux s'engendrant réciproquement comme la Bouteille de K lein. $0 \mathrm{n}$ voit bien comment, dans $\mathrm{C}$ oin de chasteté, les «caresses inframinces» sont dans le limage ou le polissage, et surtout dans la galvanisation, technique éminemment duchampienne. Notons également la curiosité duchampienne pour les surfaces rasées: le sexe féminin rasé de Étant donnés... en est un excellent exemple. Par conséquent, Coin de chasteté illustre comment l'approximation se réalise optimalement dansl'emboîtement d'un plein et d'un creux. À première vue, Coin dechasteté ressemble à une dent prise dans la gencive. Et pourtant le fantasme de la vagina dentata fonctionne symboliquement. D uchamp était particulièrement sceptique devant lesinterprétations psychanalytiques de ses œuvres, ici par exemple: I'angoisse mâle devant le sexe féminin perçu comme une bouche dentée. L'implantation du moulage en plastique dans la matrice consacre, pour D uchamp, l'infra-mince, la contiguïté métonymique du 
contenant et du contenu, du convexe et du concave, du plein et du creux.

En conclusion provisoire, la fragmentarisation du corps chez D uchamp a un double effet: la focalisation sur lesfragments essentiels - sein, vulve, verge- et la réduction de la différence sexuelle à une topologie de l'infra-mince, apologie de l'hermaphrodisme.

\section{Indices fautifs et prothèse agonistique}

L'art de M arcel D uchamp, c'est direla sémio-érotique de Rrose Sélavy, est indiciel. Aucun objet de la nébuleuse duchampienne n'est symbolique ni iconique. D uchamp exalte l'enchaînement des différences infra-minces, la métonymisation des concepts, la contiguiitédes matières. M éfiance de l'allégorie, du symbolisme. Le signifiant ne signifie pas par convention comme le langage "sérieux», l'art à message ou l'art expressif, mais il signifie par la chance des associations. Ainsi signifie cette série ininterrompue de cal embours duchampiens. L'art ne raconte rien, ne renvoie à rien, il syntagmatise le convexe et le concave, le plein et le creux, la verge et la vulve selon la topologie de l'infra-mince. Rien de symbolique, par conséquent, aucun renvoi à un signifié par un signifiant arbitraire. Rien d'iconique non plus puisque aucune ontologie de référents n'inviteà la mi mésis. II n'y a rien à représenter selon la noble manera et avec lebuon gusto de I'Art-majuscule puisque l'essentiel est ready-made. Reste I'indice. L'art est l'empreinte de la vie: s'il y a distance entre art et vie, ellene peut êtrequ'infra-mince. Comme $O$ bjet-D ard est l'indice de F euille de vigne femelle. L'artminuscule de Duchamp est un champ d'indices. C'est sur le fonds du corps-corps que les fragments de chair essentiels s'indexicalisent réciproquement. Le geste artistiqueest une opération d'indexicalisation selon la topologie de l'infra-mince, et nous voudrions illustrer trois stratégies duchampiennes d'indexicalisation: I'appropriation, la coupure, la prothèse, dans I'ordre d'une dramatisation croissante puisque la prothèse chez $\mathrm{D}$ uchamp, on le verra, pointe vers la mort.

On peut aller vite sur la stratégie d'appropriation du «ready-made rectifié» qu'est L.H . O.O.Q. ( «elle a chaud au cul »), de 1919, date vers laquelle Freud démontrait I'homosexualité de Leonardo. Profanation dadaïste ou pas, la M ona Lisa devient victime d'une appropriation.
La transposition que $D$ uchamp effectue fait transgresser le seuil de la proximité infra-mince du féminin et du masculin: conjunctio oppositorum par quelques traits de crayon, appropriation d'une œuvre d'art parmi les plus sublimes par un Duchamp qui, déjà à cette époque du Grand Verre, est saisi par la topologie de l'infra-mince. L.H.O.O.Q. illustre une transposition qui indexicalise l'infra-mince sexuel, par conséquent le corps essentiel.

Stratégie d'indexical isation plus dramatique, celle de la coupure Vingt exemplaires de La Boîte en valise contiennent le Paysage fautif [ill. 16], et huit autres cette constellation sans titre [Poils coupés] [ill. 17]. Coupure au senslittéral du terme: des poils de la tête, d'en dessous des aisselles et des poils pubiens sont collés sur un rectangle de plastique. Cette composition suggestive sert d'indice du corpsféminin. Paysagefautif est un rectangle detoile noire, décolorée partiellement par les restes séchés de sperme. $\mathrm{Ce}$ «paysage» étrangea pris de belles couleurs oxydées, et il ne représente pas une «faute» plus grave que celle d'une masturbation dans le jeu érotique ${ }^{19}$. Coupure des poils, jet de sperme, autre stratégie d'indexicalisation pointant vers le corps essentiel.

Stratégie d'indexicalisation sans doute culminante, celle de la prothèse puisque l'infra-mince règle ici maximalement la topologie signifiante. With my tongue in my chedk (1959) [ill. 18] est une expression idiomatique en anglaisqui signifiequel'on parlesans l'intention d'être vraiment sincère. L'humour, voirel'ironie, ne peut masquer le statut agonistique de cet autoportrait 20 . G eorges D idi-H uberman y voit surtout un «malaise dans la représentation», la brutalité en tant que caractéristique essentielle de l'ironie duchampienne ${ }^{21}$. II nous semble que l'on peut aller plus loin sans tomber dans une interprétation trop facilement existentielle. With my tongue in my cheeck, en effet, est un autoportrait d'un homme de soixante-douze ans, bas-relief fabriqué de plâtre sur papier avec dessin au crayon, monté sur bois. Plâtre encore de la prothèse comme le plâtre de la F euille de vigne femelle, de l'O bjet-D ard et du Coin de chasteté. D uchamp met littéral ement la langue dans la joue et il la fait ainsi gonfler. Le plâtre est un moule qui remplit la jouecreusedu vieil homme et lui donneun relief exagéré. Il s'agit bien d'un masque funéraire mais I'œil ouvert - qui n'aurait pas pu être plâtré- marque encore la vie bien queleregard soit fossilisé. L'apparenceest mortuaire, 
également dansl'absence de couleur, marcheversla mort d'un toujours vivant. La prothèse en plâtre génère une double signifiance. $D$ 'une part, elle remplit un manque, I'absence de joue, I'absence d'un corps en vie, mais, d'autre part, par sa blancheur elle semble «manger» la vie en progressant tentaculairement jusqu'à ce que toute la tête soit plâtrée, jusqu'à ce que le masque funéraire soit complet. La prothèse préservel'illusion de vieà travers l'image de la mort. Par conséquent, est figuré ici le seuil de la vie devant la mort, l'empreinte de la mort dans la vie, le moulage inexorable de la vie par la mort. L'écart vie-mort est infra-mince. $0 \mathrm{n}$ est en plein dans la sémioérotique duchampienne, sans doute dans sa culmination. La progression agonistique gagne et la «prothétisation » secomplète. D uchamp superviseuneannéeavant sa mort, au printemps 1967, I'assemblage d'un masque funéraire en bronze soutenu par un brasimplantédans un fragment de son jeu d'échecs favori [ill.12]. Le sculpteur Alfred W olkenberg avait moulé le visage et le bras droit de D uchamp à cette fin. U n cheval d'échecs assiste, la tête courbée, à cette mécanisation, cette fragmentarisation, cette indexicalisation de la fin.

\section{G loire des empreintes}

L'infra-mince se réalise par excellence par le moulage. T out dynamisme créateur d'ailleurs est placé par Duchamp sous le signe de l'empreinte, de la trace, du moulage22. Personnemieux que G eorges D idi-H uberman a étudié l'exigence de l'empreinte chez Duchamp. Le vocabulai re del'empreinte est omniprésent dans les $\mathrm{N}$ otes duchampiennes, tout au long des années. $M$ ais plus important est que le paradigme duchampien du moule suppose, dans la pratique artistique, la production d'une forme par contact. Ainsi l'empreinte-mouvement, I'inscription mouvante est celle de la photographie et de l'imprimerie. Le «technicien bénévole», l'artisan qu'est l'artiste, est avant tout un imprimeur. Lechamp technique du métier méticuleux et précis d'artiste exige de l'expérience et une heuristique des supports et matériaux.
Le hasard et la précision s'associent dans la production del'empreinte. L'empreinte donnenaissanceà des formes mais elle produit en même temps un renversement de sens de cesformes. II est vrai quel'empreintene témoigne que de la quasi-réversibilité des choses: la ressemblance de contact qui marque n'importe quelle empreinte nous impose un écart, infra-mince il est vrai mais infra-mince quand même. La «précision tactile», même si elle mène idéalement à l'enchâssement ou à l'interpénétration, comporte une dimension d'indifférence, l'indifférence de l'écart. Le semblable tient au différent et le différent au semblable. M ais produire le semblableest produirel'écart, le dissemblable à lui-même, le même comme négativité. Si on peut oser une esquisse de l' «ontologie» de D uchamp, ce serait bien celle-là: «un, c'est l'unité, c'est la mêmeté close. D eux, c'est la dualité, c'est I'opposition sèche. II faut donc ajouter le "trois", c'est-à-direl'ouverture dialectique de la différence» 23 .

Concluons. La pratique duchampienne met en scène, avec allégresse toujours, le concept du corps essentiel, de sa mécanique, de ses fragments, de ses indices. Le désir pousse la vie vers la mort. II y a de l'impuissance dans le regard du voyeur et dans la pulsion desC élibatai res. Vivre la quatrième dimension n'est pas pour aujourd'hui. La $M$ ariée s'esquive et la fusion n'est qu'un fantasme. $N$ ous vivons la topologie de l'infra-mince, dans la rencontre avec I'Autre, dans la rencontre avec la mort. Écart inframince il y aura toujours. L'art ne témoigne pas du désir ni de la mort, mais de l'écart infra-mince entre moi et I'Autre dans le désir, l'écart infra-mince entre la vie et la mort. La mort est la prothèse de la vie, c'est ce que nous laisse voir W ith my tonguein my cheek. L'art deD uchamp, d'après D uchamp, s'installedansl'infra-mincequi sépare Éros et Thanatos, s'installe dans l'infra-mince espace qui sépare la mort de la vie, toujours, quand même. 


\section{NOTES}

1. The Complete W orks of M arcel D uchamp de A. Schwartz (N ew York, D elano G reenidge Ed., 1997; 3e éd. revue et augmentée) est un outil de travail indispensablebien queletexte deSchwartz, qui sert d'introduction au catalogue raisonné, est souvent assez spéculatif. À part les classiques sur D uchamp (R. Lebel, T. de D uve, J. Suquet, F. N aumann et J. Clair, entre autres), le M arcel D uchamp de D. Ades, N . C ox et D. H opkins (Londres, Thames \& H udson, 1999) est une excellente introduction à D uchamp. U ne sélection de la correspondance de $D$ uchamp vient d'être publiée par F. M . N aumann et H . O balk sous letitre deAffectionately, M arcel ( $G$ hent/ Amsterdam, Ludion, 2000). Les lettres publiées ne comportent que peu d'éléments substantiels pour la compréhension del'œuvre de D uchamp et elles ajoutent surtout des suppléments d'information concernant la biographie intellectuelle de D uchamp.

2. D. Judovitz, dans U npacking D uchamp: Art in T ransit (Berkeley/Los Angeles/London, U niversity of California Press, 1995), expose en profondeur les motifs et les effets de cette décision radicale de $D$ uchamp de ne plus peindre et elle n'hésite pas à en donner une explication psychanalytique. Le livre de Judovitz, dans la masse de littérature secondaire sur D uchamp depuis dix ans, se distingue par son point de vue original et globalisant.

3. R. D adoun, D uchamp, cemécano qui met à nu, Paris, $\mathrm{H}$ achette, 1996, p. 79.

4. «En général, si cemoteur mariéedoit apparaîtrecommeuneapothéose de virginitéc'est-à-dire le désir ignorant, le désir blanc (avec une pointe de malice) et s'il (graphiquement) n'a pas besoin de satisfaire aux lois de l'équilibre pesant, néanmoins, une potence de métal brillant pourra simuler l'attachedela pucelleà sesamieset parents. [...] T outel'importance graphique est pour cet épanouissement cinématique. [...] Cet épanouissement cinématique est commandé par la mise à nu électrique. [...] D ans cet épanouissement, la mariée se présente nue sous deux apparences: la première, celle de la mise à nu par les célibataires, la seconde apparence, celleimaginative-volontaire dela mariée». (M . D uchamp, dans D uchamp du signe, Paris, Flammarion, 1994, p. 62-63).

5. Ibid., p. 62

6. Je dois cette information àJ. A. Ramirez, D uchamp. Love and D eath, Even, London, Reaktion Books, 1998, p. 139. Lelivre de Ramirez poseles questions essentielles à propos de D uchamp.

7. Cette remarque superbe est faire par J. Suquet, $M$ arcel D uchamp ou l'ébloui ssement de l'éclaboussure, Paris, L'H armattan, 1998, p. 77.

8. J. Clair développe l'idée que Duchamp va ainsi à l'encontre du naturalisme, qu'il veut faireun «art non rétinien » et qu'il selaisse inspirer à ce propos par les découvertes de la radiographie, par des études de physique concernant la magnétisation et par les possibilités de la photographie(D uchamp et la photographie. Essai d'analysed'un primat technique sur le développement d'une œuvre, Paris, Éd. D u Chêne, 1977, p. 14-25). 9. T. de D uve, dans Résonances du ready-made. D uchamp entre avantgarde et tradition (N îmes, Chambon, 1989), est parmi ceux qui estiment que Étant donnés.. constitue plutôt une régression dans l'œuvre de D uchamp. D ans Le Grand Verre, «D uchamp imagine la rencontre de I'objet et du public à la manière de cette rencontre manquée, manquée du moins pour les célibataires enchainés dans les trois dimensions del'espace, mais qui réussirait si le saut dans la quatrième dimension était possible» (p. 33), mais Étant donnés... n'ouvre même plus cette possibilité de la quatrième dimension : le regardeur y est confronté bilatéralement et en solipsiste avec l'objet réel à trois dimensions, et pourtant «l'art a lieu dans la quatrième dimension où l'épanouissement horizontal volontaire de la mariée va à la rencontre de l'épanoui ssement vertical de la mise à nu par ses célibataires et produit l'épanouissement vertical par conciliation» (p. 39). Épanouissement et quatrième dimension sont intrinsèquement liés. J.-F. Lyotard, dans L es T ransformateurs D uchamp (Paris, Galilée, 1977), cite à ce propos D uchamp lui-même, dans un entretien avec $A$. Schwartz, et il commente ainsi : «[...] M onsieur $M$ arcel se travestit en $M$ lle Rrose et travailleles "coupures". Passant outreàl'importancedonnéeàla différence des sexes, et donc à leur réconciliation, il va au-delà, beyond sex. "Le sexe n'est pas la quatrième dimension. II est tridimensionnel aussi bien que quadridimensionnel. $0 \mathrm{n}$ peut certes exprimer un par-delà le sexe en le transférant dans une quatrième dimension. $M$ ais la quatrième dimension n'est pas le sexe en tant que tel. Le sexe n'est qu'un attribut, il peut être transféré dans une quatrième dimension, mais il ne constitue pas la définition ou le statut de la quatrième dimension. Le sexe est le sexe". Le sexe, le premier, le deuxième, le troisième, etc., est un produit d'identification, une fiche de la police des désirs: ce que la costruzione leggitima fait des espaces passionnels» (p. 94-95).

10. J. Clair, "Sexe et topologie», dans M arcel Duchamp, l'abécédaire, Paris, Centrenational d'art et deculture Georges-Pompidou, 1977, p. 55. V oir également D. Judovitz, op. cit., p. 202-219, pour une interprétation consistante de la M ariée de Étant donnés..

11. J.-F. Lyotard donne la description suivante du punctum : «La vulve qu'on ne peut manquer de remarquer, on nevoit que ça, est dépouillée de toute fourrure (alors que les aisselles sont garnies, cen'est pas une enfant), les cuisses sont écartelées, les grandes lèvres en érection sont ouvertes, elles laissent apercevoir non seulement les petites lèvres tumescentes, mais I'orifice béant du vagin et mêmeles bulbes vestibulaires gonflés, autour de la commissure inférieure. La vulve élève la vue? 0 u : la vulvée lève la vue?»(op. cit., p. 143). 
12. A. Schwartz, op. cit., vol. 1, p. 228-229, soutient le premier point de vue, commed'ailleurs la plupart des critiques duchampiens, tandis que J. Clair (art. cit., p. 56-59) soutient le second point de vue. Je penche plutôt vers l'interprétation de J. Clair.

13. J. Clair, art. cit., p. 56.

14. Clair sedit d'accord avec Schwartz pour insister sur I'hermaphrodisme comme thème essentiel de l'œuvre de D uchamp. «Le modèle vient des géométries non euclidiennes [...] La transsexualitéchez D ucamp - son jeu sur letravesti, qui va de R roseSélavy jusqu'au (defaçon mineuremaisaussi significative) Couple de tabliers (des manchons qui peuvent se retourner comme des doigts degant) - , est une sorte d'expérience ontologique naïve d'une idéalité mathématique où s'abolit la différenciation sexuelle» (dans Sur M arcel D uchamp et la fin de l'art, Paris, Gallimard, 2000, p. 168). 15. J. Clair cite, dansD uchampet la photographie, un entretien deD uchamp avec D. de Rougemont en 1945 où il précise cette notion d'infra-mince: «[C'est quelquechose] qui échappeànos définitionsscientifiques. ]'ai pris à dessein le mot mince qui est un mot humain, affectif, et non pas une mesure précise de laboratoire. Lebruit ou la musique quefait un pantalon develours côtelécommecelui-ci, quand on bouge, relèvedel'infra-mince. Le creux dans le papier, entre le recto et le verso d'une feuille mince... À étudier ! ... C'est une catégorie qui m'a beaucoup occupé depuis dix ans» (p.96). Et J. Clair de commenter: «L'infra-mince serait ainsi le degré qualitatif où le même se transforme en son contraire, sans qu'on puisse exactement décider qui est encore le même et qui est déjà l'autre. $D$ 'un point de vue purement géométrique, on pourrait dire que c'est la notion qui fait intervenir le passage à la limite. [...] $M$ ais du point de vue plus sensible, plus intuitif, on pourrait dire que l'infra-mince est la lisière infiniment mince qui définit un seuil: seuil d'audition, seuil de vision, seuil d'odorat, tout ce qui ressortit au plus aiguisé de la sensation. La touche imperceptible, infinitésimale que le sculpteur - un Brancusi, par exemple - donneà une courbe pour obtenir l'effet désirérelève del'inframince. Aussi bien Duchamp définit-il encore l'infra-mince comme la différenceentrelecreux d'un mouleet leplein du moulagecorrespondant » (p. 98).

16. M. D uchamp, N otes, Paris, Flammarion, 1999, p. 19-47.

17. G. Didi-H uberman, L'Empreinte, Paris, Éd. du Centre-Pompidou, 1997, p. 167.
18. LesN otescomportent un passageoù $D$ uchamp évoquecetteprogression dansl'approximation : «Limage - polissage - La lime infra-mince - papier deverre- toileémeri, ponçage du laque, souvent ces opérations atteignent à l'infra-mince. C oupage - coupant (passicot, lames de rasoir), glissage séchage - collage, viscosité - cassage. Brûlage, fondage [...] Porosité imbibage (papier buvard). Perméabilitéà l'eau et à l'air (cuir). Enfonçage (clous, plantedeflèche), frottage, grattage- ajustage, repérage (camouflage, retissage - ou réparation mécanismique). Adhérence, collage - Empesage - Caresses infra-minces» (N otes, 26-28, 27).

19. A. G ervais noteà propos de Paysage fautif que «D uchamp ira jusqu'à inscrire au cœur même du seul isme qui lui soit cher, l'érotisme, au cœur même d'un seul acte, I'acte d'amour» (C'est. M arcel Duchamp dans «la fantai sie heureuse del'histoire», N îmes, Chambon, 2000, p. 291). V oici la description del'œuvre: «à usage privé, est fait despermesur astralon, tissu transparent, lui-même sur satin noir, tissu opaque» (p. 289). Gervais reconstruit tout l'intertexte et les jeux de mots autour de Paysage fautif. 20. Voir D. Judovitz, op.cit., p.114-119, pour une interprétation psychanalytique de With my tongue in my cheek. Elle voit également une relation originale avec l'intérêt que $D$ uchamp portait à la numismatique, interprétant W ith mytonguein my cheek commeunemédaille «progressante» (p. 185-194)

21. G. Didi-H uberman, op.cit, p. 173.

22. G. W ajcman généralise «cettetechniqueartistiquepratiquederien du tout » qu'est le moulage: le moulage est capable de «faire surgir une figure logique absolument bouleversante, qui est le Rien positivé», I'Absence comme 0 bjet ( L' $O$ bjet du siècle, Paris, V erdier, 1998, p. 149-150). «N ous serions conduits à ceci que ces œuvres, ces objets qui recueillent l'absence de l'objet qui les complète sont, en somme et tout bien considéré, des moules, desmoulesnégatifs, dansquoi secoulelemanque, qui devient ainsi la forme "positive", l'objet moulé. D'où l'idée qui prend forme de considérer Fountain commeunepremièreversion, au moinsun antécédent logique de la Feuille de vigne femelle, moulage d'un sexe féminin, ce qui restitue un creux en relief montagneux, le positif érigé d'une fente. Bien sûr, c'est un chibre, façon 0 bjet-D ard, qui vient d'abord à l'esprit comme le complément absent, naturel, dela F ountain » (p. 84). H ypothèse hardie mais séduisante, il nous semble.

23. G. Didi-H uberman, op.cit., p. 163. 


\section{Les illustrations de Marcel Duchamp}

1. Vierge $n^{\circ} 2,1912$. Philadelphia Museum of Art, Philadelphie.

2. Le Passage de la Vierge à la Mariée, 1912. Museum of Modern Art, New York.

3. Mariée, 1912. Philadelphia Museum of Art, Philadelphie.

4. Le Grand Verre ou La Mariée mise à nu par ses célibataires, même, 1915-1923. Détail du Pendu femelle. Philadelphia Museum of Art, Philadelphie.

5. Valve Auvard, tirée du catalogue Hartmann de 1911

6. La Mariée mise à nu..., 1968. Collection particulière, Paris.

7. Portrait du Docteur Dumouchel, 1910. Philadelphia Museum of Art, Philadelphie.

8. Torture-morte, 1959. Musée national d'art moderne, Paris.

9. Prière de toucher, 1947. Couverture pour le catalogue de l'Exposition Internationale du Surréalisme à la Galerie Maeght, Paris.

10. Feuille de vigne femelle, 1950. Collection particulière, Paris.

11. Objet-Dard, 1951. Collection particulière, Paris.

12. Autoportrait de Duchamp, 1968. Masque funéraire de Duchamp réalisé par le sculpteur Alfred Wolkenberg (moulage du visage et du bras droit de Duchamp).

13. À la manière de Delvaux, 1942. Collection Veras et Arturo Schwarz, Milan.

14. Le gaz d'éclairage et la chute d'eau, 1948-1949. Moderna Museet, Stockholm. Étude préliminaire de Étant donnés : $1^{\circ}$ la chute d'eau, $2^{\circ}$ le gaz d'éclairage, 19461966.

15. Coin de chasteté, 1954. Collection particulière, Paris.

16. Paysage fautif dans La Boîte en valise, 1936-1941. Musée national d'art moderne, Paris.

17. Sans titre [Poils coupés] dans La Boîte en valise, 1936-1941. Musée national d'art moderne, Paris.

18. With my tongue in my cheek, 1959. Musée national d'art moderne, Paris.

(๔ Succession Marcel Duchamp / SODRAC (Montréal) 2000 

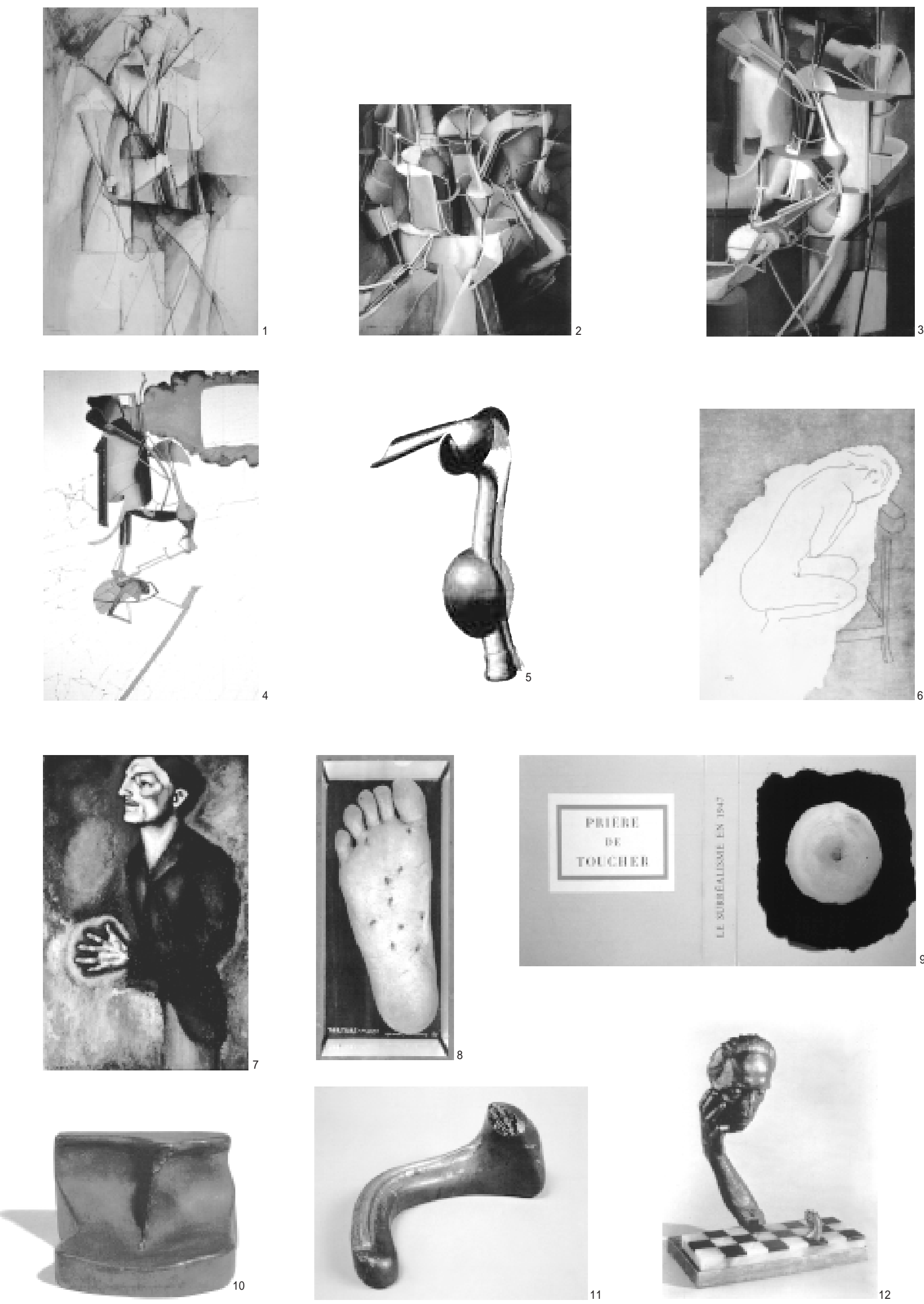

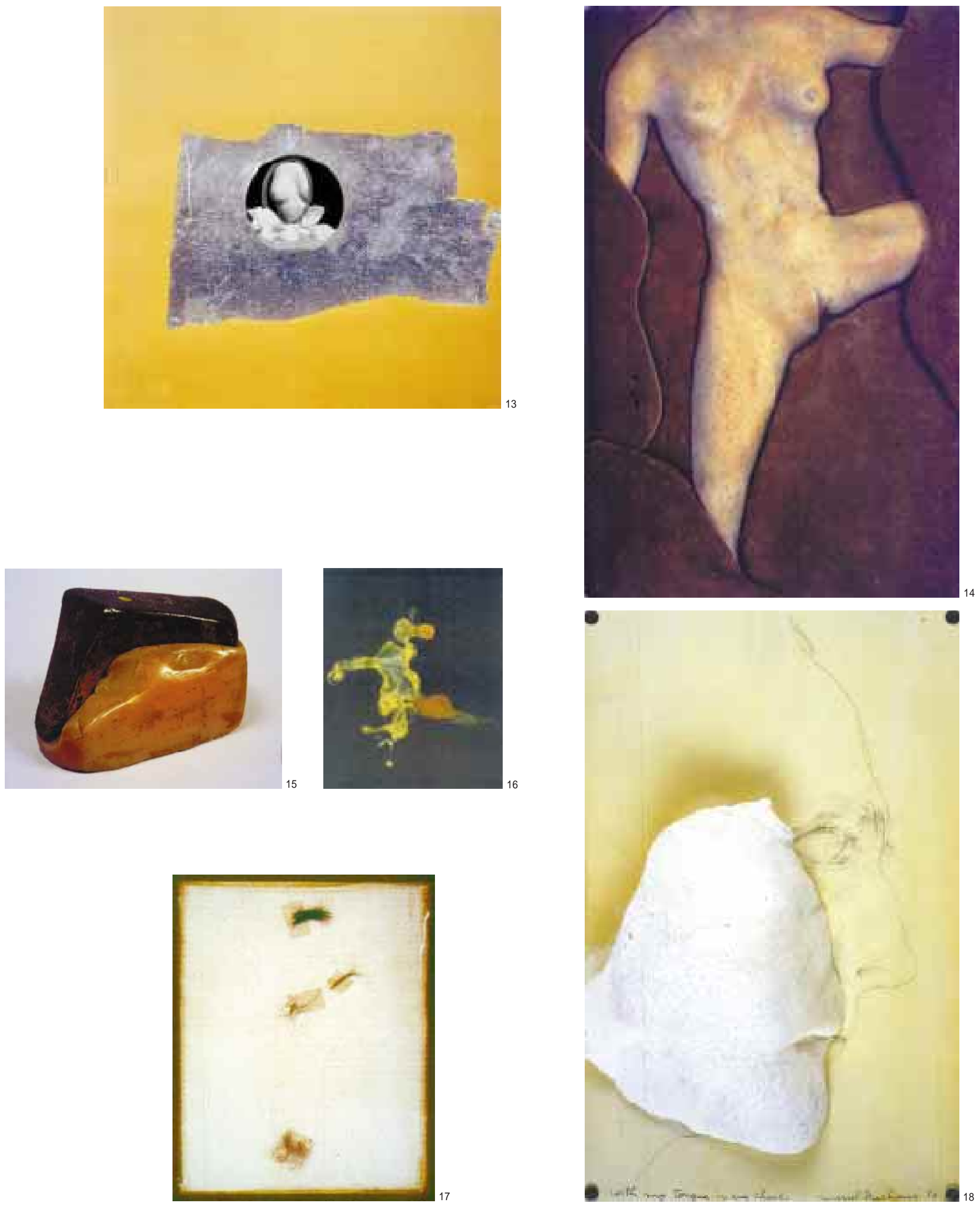